\title{
The Better Understanding and Recognition of the Disconnects, Experiences, and Needs of Patients with Chronic Idiopathic Constipation (BURDEN-CIC) Study: Results of an Online Questionnaire
}

\author{
Lucinda A. Harris • John Horn • Michele Kissous-Hunt • Leslie Magnus • \\ Eamonn M. M. Quigley
}

Received: July 21, 2017 / Published online: November 6, 2017

(C) The Author(s) 2017. This article is an open access publication

\section{ABSTRACT}

Introduction: There is limited literature comparing the experiences and attitudes of patients with chronic idiopathic constipation (CIC) to those of healthcare professionals (HCPs) treating CIC patients. The BURDEN-CIC study was conducted to better understand the experiences and ongoing needs of CIC patients and to assess their alignment versus disconnection with the perceptions and needs of HCPs who treat CIC patients.

Methods: The BURDEN-CIC study was an author-developed, online questionnaire that used KnowledgePanel ${ }^{\circledR}$ to survey individuals with CIC $(n=1223)$. HCPs who treat CIC patients were recruited separately and

Enhanced content To view enhanced content for this article go to http://www.medengine.com/Redeem/ A8CCF0603C6A861B.

L. A. Harris

Mayo Clinic, Scottsdale, AZ, USA

J. Horn

University of Washington, Seattle, WA, USA

M. Kissous-Hunt

Mount Sinai GI, New York, NY, USA

L. Magnus

Synergy Pharmaceuticals Inc, New York, NY, USA

E. M. M. Quigley ( $\square)$

Houston Methodist Hospital, Houston, TX, USA

e-mail: equigley@houstonmethodist.org participated in a complementary online questionnaire $(n=331)$.

Results: Most patients had used (58\%) or were using (51\%) over-the-counter treatments for their CIC, with only $16 \%$ currently on prescription therapy. More than half (59\%) of current CIC prescription users were not satisfied/completely satisfied with their current chronic treatment. Many patients $(42 \%)$ felt frustrated regarding their CIC, and a similar percentage (40\%) expressed acceptance that CIC was part of their daily life. The majority of HCPs agreed that CIC patients were frustrated (72\%), stressed (50\%), or fed up (43\%) with current treatment options but were relatively unaware $(21 \%)$ that patients were accepting of their CIC. HCPs reported the greatest challenges in treating CIC patients as response rates to current therapies (55\%), treatment adherence (55\%), management of treatment-related diarrhea (34\%), and lack of treatment options (34\%).

Conclusion: BURDEN-CIC identified that many patients and HCPs are frustrated and not satisfied with current CIC treatments due to lack of efficacy and side effects, such as diarrhea. The survey identified that many patients are "accepting" of their disease, potentially compromising treatment outcomes. More dialogue is needed between HCPs and CIC patients, especially regarding management of treatment expectations and side effects. Further, additional treatment options would be useful for both patients and HCPs.

Funding: Synergy Pharmaceuticals Inc. 
Keywords: Chronic idiopathic constipation; Functional constipation; Gastroenterology; Quality of life; Survey

\section{INTRODUCTION}

Chronic idiopathic constipation (CIC) is a common functional gastrointestinal disorder whose prevalence ranges from $2 \%$ to $27 \%$ among adults, averaging $14 \%$ in the United States (US), and this percentage rises with increasing age $[1,2]$. Chronic constipation has been traditionally defined by the frequency of bowel movements. Guidelines set forth by Rome IV diagnostic criteria base the diagnosis on patient-reported symptoms such as straining, lumpy/hard stool consistency, and the sensation of incomplete bowel movements [3]. Rome IV criteria also identify that CIC can be accompanied by the presence of abdominal symptoms such as bloating and discomfort [3]. Additionally, many patients report that CIC adversely affects their quality of life and increases overall healthcare costs $[4,5]$.

The current treatment goal is to provide multi-symptom relief. The treatment of constipation traditionally begins with increased dietary fiber and supplementation with bulking agents, exercise, and bowel habit training [3]; however, often only partial relief is obtained, and many patients use laxatives on a regular basis without medical supervision [6]. Laxatives available over the counter (OTC) are generally recommended for episodic and not chronic use $[7,8]$. If the response to OTC medications is deemed inadequate then prescription pharmacotherapies are the next line of treatment. At the time this study was conducted, MiraLAX ${ }^{\circledR}$, lactulose, linaclotide (Linzess $^{\circledR}$ ), and lubiprostone $\left(\right.$ Amitiza $\left.{ }^{\circledR}\right)$ were available for prescription treatment of CIC.

A number of patient-based surveys have reported that patients remain concerned and dissatisfied with traditional, OTC, and then available prescription laxatives [e.g., polyethylene glycol 3350 (PEG; MiraLAX ${ }^{\circledR}$ ), lactulose] for CIC, leaving an unmet need to be addressed by new and differentiated treatment options $[4,9,10]$. There is, however, limited published literature comparing the experiences and attitudes of patients with CIC to the understandings and perceptions of healthcare professionals (HCPs) who treat CIC patients. The better understanding and recognition of the disconnects, experiences, and needs of patients with chronic idiopathic constipation (BURDEN-CIC) study was designed to better understand the management pathway, overall satisfaction with care, impact on quality of life, and unmet treatment needs for patients with CIC, from the perspectives of both patients and the HCPs who treat CIC patients.

\section{METHODS}

\section{Patient Questionnaire}

The questionnaire for the BURDEN-CIC study was developed by all the authors, with the patient protocol and associated materials reviewed and approved by the Western Institutional Review Board. The BURDEN-CIC study utilized a proprietary consumer panel (KnowledgePanel ${ }^{\circledR}$ ) in conjunction with an additional opt-in panel to identify individuals suffering with CIC. KnowledgePanel ${ }^{\circledR}$ is a representative sample of the total US population that accounts for demographic and epidemiological factors, including age, race, marital status, education, employment status, and geographic region. Recruitment and surveying were conducted between June 29, 2016, and January 30, 2017. The questionnaire was self-administered over the Internet and potential participants were told only that they were answering a questionnaire about their health when initially being screened for participation. Eligible panelists received notification of their assigned questionnaires through a password-protected e-mail account. All questionnaire responses were confidential, with any identifying information removed. Participants had the option to leave the survey at any time, and any incomplete study data were removed from the overall database. Demographic and geographic distributions from the Current Population Survey were used as benchmarks [11]. 


\section{Inclusion and Exclusion Criteria}

Panel members who agreed to participate in the study were asked to complete an online, self-administered, six-question screener to evaluate whether they met entry criteria. Respondents were eligible to participate in the BURDEN-CIC study if they were proficient in the English language, were aged $\geq 18$ years, had been diagnosed with CIC or fulfilled Rome IV criteria for CIC, had not been previously diagnosed as having irritable bowel syndrome with constipation (IBS-C), and had not been previously diagnosed as having any disease of organic cause with associated constipation (e.g., gastrointestinal, neurological). Thus, there were two groups of CIC respondents: one with participants who had a formal diagnosis of CIC from an HCP and another with participants who were undiagnosed but who fulfilled Rome IV criteria for CIC from the screener.

Potential participants were not eligible to take the BURDEN-CIC patient questionnaire if they were currently pregnant or had been pregnant in the past 12 months. Respondents were specifically asked whether they had been diagnosed by an HCP as having cancer, an organic gastrointestinal disease (e.g., diverticulitis, diverticulosis, inflammatory bowel disease), IBS-C, or spastic or irritable colon. Participants who reported abdominal pain-the hallmark symptom of IBS-C-were not excluded from the BURDEN-CIC study unless they indicated having been diagnosed with IBS-C. Respondents who had never been diagnosed with IBS-C but met Rome IV IBS-C criteria as determined from the screener, and those who had been taking opioids or any treatments not approved by the US Food and Drug Administration for CIC at the time of the study, were also excluded.

\section{Questionnaire Administration}

Participants were required to read and understand an informed consent document and were then given the option to continue with the online questionnaire. If any panelist did not provide informed consent, they were not able to participate. Panelists who met the inclusion criteria completed an online, self-administered questionnaire that was estimated to take $45 \mathrm{~min}$ to complete. Panelists were advised that their participation was voluntary and that they had the option of not answering specific questions. As part of the online study, participants were asked to report the symptomatology of their CIC, the impact of constipation symptoms on activities of daily living, effects and side effects of CIC treatments, and treatment satisfaction. Question and answer formats included dichotomous, multiple-choice, and openended, as well as numeric rating scales (1-5 or 1-7). Data were internally validated to ensure that patients completed the questionnaire only once, that sufficient time was spent completing the questionnaire, and that there was a consistent response pattern.

\section{Healthcare Professional Questionnaire}

For the HCP questionnaire, healthcare prescribers who actively treat patients with CIC were recruited to participate in a 45-min online questionnaire based on the IRB-approved Patient Questionnaire, which was fielded from July 20 to August 10, 2016. The questionnaire was targeted to a random national sample of gastroenterologists (GEs), primary care physicians (PCPs), nurse practitioners (NPs), and physician assistants (PAs). To be eligible to participate, an HCP must have been actively managing patients with CIC, seeing at least 50 total patients and at least 15 CIC patients per month. No geographical or workplace setting criteria were implemented. HCPs were recruited independently of participants in the Patient Questionnaire and were remunerated for their participation in the study. Question and answer formats included dichotomous, multiple-choice, and open-ended, as well as Likert rating scales (1-5 or $1-7)$.

\section{Ethical and Legal Aspects}

The patient protocol and associated materials were reviewed and approved by the Western Institutional Review Board. Both the patient and HCP questionnaires were conducted by GfK according to the globally accepted standards of good clinical practice (as defined in the 
International Conference on Harmonisation E6 Guideline for Good Clinical Practice), in accordance with the Health Insurance Portability and Accountability Act, and in keeping with local regulations. Informed consent was obtained from all respondents for being included in the study.

\section{Statistical Analysis}

A standardized statistical analysis approach was undertaken across both studies with summary statistics such as mean values, standard deviations, minimums, quartiles, and maximums for continuous variables and counts and percentages for categorical variables. Additional analyses were conducted with respondents who were felt to have factors that would characterize them as having "more severe" CIC. These respondents had $\geq 1$ of the following:

- Productivity impacted by CIC symptoms (defined as $\geq 1$ day in a typical month where CIC symptoms interfered with productivity), or

- Personal activity impacted by CIC symptoms (defined as $\geq 1$ day in a typical month where CIC symptoms interfered with personal activity), or

- An emergency room (ER) visit for CIC-related symptoms (defined as $\geq 1$ visit in the past year), or

- A current prescription treatment for CIC (defined as $\geq 1$ prescription for linaclotide, lubiprostone, PEG, senna, or lactulose/ kristalose).

\section{RESULTS}

There were 29,665 clicks on the Patient Questionnaire link, with 27,709 panelists completing the screening test. A total of 1223 respondents qualified for and completed the questionnaire. The majority of respondents were female $(69 \%)$, Caucasian (non-Hispanic; $65 \%$ ), and married (55\%), with a mean age of 49.1 years (Table 1 ). Patients had suffered from symptoms of CIC for a median of 4 years (mean age at symptom onset, 44.9 years).
Table 1 CIC patient demographics and baseline characteristics

\begin{tabular}{ll}
\hline & $\begin{array}{l}\text { CIC patients } \\
n=1223\end{array}$ \\
\hline Female & $69 \%$ \\
Age, years, mean (SD) & $49.1(18.0)$ \\
Age at symptom onset, years, mean (SD) & $44.9(17.7)$ \\
Race/ethnicity & \\
White/non-Hispanic & $65 \%$ \\
Black/non-Hispanic & $13 \%$ \\
Mixed/non-Hispanic & $3 \%$ \\
Other/non-Hispanic & $3 \%$ \\
Hispanic & $16 \%$
\end{tabular}

Highest education level

High school-no graduation $\quad 7 \%$

High school—diploma/GED 27\%

College-no graduation 26\%

College-graduation $\quad 28 \%$

College-post-graduate $\quad 12 \%$

Current treatment to manage CIC symptoms

Lifestyle changes

General diet changes $\quad 32 \%$

Increased activity and exercise $\quad 24 \%$

Meditation/yoga/relaxation techniques 6\%

Gluten-free diet $\quad 5 \%$

OTC treatment remedies

Fiber $\quad 31 \%$

Stool softeners $\quad 25 \%$

Probiotics/prebiotics 20\%

Non-prescription laxatives $\quad 17 \%$

Non-prescription stimulant laxatives $\quad 17 \%$

Other $\quad 5 \%$

Prescription therapy 16\%

OTC over-the-counter 
Table 2 Healthcare professional demographics

\begin{tabular}{lllll}
\hline & GE & PCP & NP & PA \\
& $\boldsymbol{n}=\mathbf{1 5 5}$ & $\mathbf{n = 7 6}$ & $\mathbf{n = 5 0}$ & $\mathbf{n = 5 0}$ \\
\hline Male & $84 \%$ & $75 \%$ & $8 \%$ & $34 \%$ \\
Age, years, mean (SD) & $48.8(8.2)$ & $50.7(5.9)$ & $48.6(7.6)$ & $44.3(9.0)$ \\
Years in clinical practice, mean (SD) & $17.3(7.4)$ & $20.2(6.1)$ & $15.0(5.5)$ & $16.2(7.2)$ \\
Time spent in direct patient care, \%, mean (SD) & $96.5 \%(5.1 \%)$ & $97.6 \%(4.2 \%)$ & $97.0 \%(5.6 \%)$ & $96.4 \%(5.6 \%)$ \\
Practice setting & & & & \\
Community practice & $62 \%$ & $57 \%$ & $34 \%$ & $40 \%$ \\
Solo practice & $21 \%$ & $32 \%$ & $30 \%$ & $48 \%$ \\
Hospital-based practice & $9 \%$ & $8 \%$ & $18 \%$ & $10 \%$ \\
Academic practice & $8 \%$ & $3 \%$ & $8 \%$ & - \\
Medical specialty & & & & $34 \%$ \\
Primary care/general practice & - & $100 \%$ & $38 \%$ & $53 \%$ \\
Gastroenterology & $100 \%$ & - & $38 \%$ & $12 \%$ \\
Internal medicine & - & - & $18 \%$ & \\
\hline
\end{tabular}

$G E$ gastroenterologist, $N P$ nurse practitioner, $P A$ physician assistant, $P C P$ primary care physician

The online HCP questionnaire was completed by 331 HCPs, including 155 GEs, 76 PCPs, 50 NPs, and 50 PAs (Table 2). The majority of HCPs were male (63\%), had been in clinical practice for more than 17 years (mean, 17.5 years), and were spending over $95 \%$ of their time in direct patient care (mean, 97\%).

\section{Describing Constipation}

A wide variety of emotive terms were used to describe how patients feel about their CIC and its symptoms (Table 3 ). From a multiple-choice menu, respondents most often indicated feeling frustrated, being accepting of constipation as part of daily life, and feeling stressed. Respondents with more severe CIC also reported feeling frustrated, stressed, and accepting of CIC; however, they were more likely to report feeling fed up, depressed, and embarrassed and much less likely to use a positive term such as being in control. The majority of HCPs felt that patients with CIC were frustrated, stressed, and fed up about their condition, in line with what the patients themselves reported feeling. HCPs also expressed that they thought their patients were obsessed with symptoms and were lacking answers. Only $10 \%$ of HCPs thought their patients with CIC were in control and only $21 \%$ recognized that patients were accepting of CIC, suggesting a disconnection between patients and HCPs.

\section{Symptoms of CIC}

When asked to describe the first symptoms they experienced when they began suffering from CIC (multiple-choice menu), diagnosed and undiagnosed respondents used similar terms, including straining, hard/lumpy/pebble-like stools, and abdominal discomfort (Table 4). Undiagnosed respondents also commonly identified infrequent stools as the first constipation-related symptom they experienced and were much less likely to report abdominal symptoms among the first symptoms. The majority of diagnosed and undiagnosed respondents defined, in an open-ended format, 
Table 3 Common terms used to express how patients with CIC feel about their condition

\begin{tabular}{lllc}
\hline Emotive terms & $\begin{array}{l}\text { All CIC } \\
\text { patients } \\
\boldsymbol{n}=\mathbf{1 2 2 3}(\%)\end{array}$ & $\begin{array}{l}\text { More severe CIC } \\
\text { patients } \\
\boldsymbol{n}=\mathbf{8 4 7}(\%)\end{array}$ & $\begin{array}{c}\text { All HCPs } \\
\boldsymbol{n}=\mathbf{3 3 1} \\
(\%)\end{array}$ \\
\hline Frustrated & 42 & 55 & 72 \\
Accepting: just part of my life & 39 & 33 & 21 \\
Stressed & 28 & 38 & 49 \\
Fed up & 24 & 32 & 43 \\
In control: I use treatments that help me manage my symptoms & 23 & 19 & 10 \\
$\quad$ & & & 34 \\
Embll & 18 & 24 & 34 \\
Depressed & 15 & 21 & 35 \\
Lacking answers & 14 & 18 & 7 \\
Fine: it is not a big deal & 14 & 7 & 42 \\
Obsessed by symptoms & 8 & 12 & \\
\hline
\end{tabular}

CIC chronic idiopathic constipation, HCP healthcare professional

infrequent stools as bowel movements that happen only once every $3(37 \%$ and $36 \%$, respectively) or $4-5$ days $(22 \%$ and $30 \%$, respectively).

In discussing which of these CIC symptoms finally resulted in the patient seeking medical care (multiple-choice list), diagnosed patients more commonly complained of abdominal pain/discomfort (55\%) compared with undiagnosed respondents (17\%). Conversely, the symptoms of difficulty with bowel movement (63\%) and straining (42\%) led more undiagnosed respondents to talk to an HCP compared with diagnosed patients $(52 \%$ and $33 \%$, respectively). These data suggest that the presence of abdominal symptoms may be a primary trigger for patients to finally seek medical care and get diagnosed.

When describing the degree of bothersomeness of current CIC symptoms (ordinal scale of 1-5), patients and HCPs had similar opinions. In the evaluation of the impact of CIC on quality of life, most CIC respondents rated their symptoms as bothersome, very bothersome, or extremely bothersome $(32 \%, 21 \%$, and $8 \%$, respectively). Likewise, respondents with more severe CIC and HCPs were aligned in the degree of bothersomeness on quality of life (severe CIC patients, $36 \%$, 29\%, and $11 \%$; HCPs, $26 \%, 60 \%$, and $13 \%$, respectively). Of the total CIC respondent population, the top three most bothersome symptoms were difficulty with bowel movement (56\%), straining (37\%), and abdominal pain/discomfort (39\%). HCPs were aligned with patients in identifying the most bothersome symptoms of CIC as difficulty with bowel movement (47\%), abdominal bloating/ distension (45\%), abdominal pain (38\%), and abdominal discomfort (35\%).

\section{Impact of Constipation on Activities of Daily Living}

When asked how many days per month their CIC symptoms interfered with personal activities (e.g., social gatherings, sporting events, family activities, hobbies), $60 \%$ of CIC respondents were affected on 4 out of 30 days per month, which equates to nearly 7 weeks per year. Diagnosed patients experienced an average of 4 days/month with personal activity impacted, increasing to 7 days/month for those patients who reported at least 1 day/month impacted (33\%). Undiagnosed respondents 
Table 4 Symptoms first experienced by patients suffering from CIC

\begin{tabular}{llll}
\hline Symptoms & $\begin{array}{l}\text { All CIC } \\
\text { patients } \\
n=1223\end{array}$ & $\begin{array}{l}\text { Diagnosed } \\
\text { patients } \\
\boldsymbol{n}=\mathbf{1 0 3 5} \text { (\%) }\end{array}$ & $\begin{array}{l}\text { Undiagnosed } \\
\text { patients } \\
\boldsymbol{n}=\mathbf{1 8 8}(\%)\end{array}$ \\
\hline Difficulty with bowel movement & 82 & 81 & 87 \\
Straining & 64 & 60 & 79 \\
Hard, lumpy, or pebble-like stools & 57 & 55 & 66 \\
Infrequent stools & 53 & 49 & 68 \\
Abdominal discomfort & 51 & 55 & 35 \\
Feeling of incomplete bowel movement, like you did not & 49 & 50 & 48 \\
$\quad$ "finish" & & & 38 \\
Bloating/distension & 48 & 50 & 21 \\
Stomach cramps & 33 & 36 & 20 \\
Bleeding (blood on stool, toilet paper, or in toilet bowl) & 24 & 26 & 12 \\
Rectal pain & 21 & 23 & 10 \\
Nausea & 13 & 14 &
\end{tabular}

CIC chronic idiopathic constipation

reported an average of 1 day/month with personal activity impacted, increasing to 5 days/month when including those with at least 1 day/month impacted (67\%). Patients also reported missing time from work and school: $25 \%$ of patients reported that they missed $\sim 60$ days per year, which equates to a potential annualized percentage loss of productivity of $23 \%$. Diagnosed and undiagnosed respondents averaged 5 and 3 days/month, respectively, where productivity was impacted due to CIC. Finally, 14\% of patients visited the ER with a median occurrence of one visit per year, which provides an increased burden on hospital and emergency care resources. GEs reported that $16 \%$ of CIC patients who are under their care visited the ER because of their constipation symptoms, which is in close alignment with what patients reported.

\section{Treating the Symptoms of CIC}

Prior to seeing an HCP, both diagnosed and undiagnosed respondents first turned to dietary changes $(45 \%$ and $37 \%$, respectively), increased fiber (40\% and $42 \%)$, and stool softeners $(37 \%$ and $35 \%)$ to manage their symptoms of CIC. Patients also cited using OTC laxatives (24\% and 27\%) and OTC stimulant laxatives (24\% and $25 \%$ ). In total, $40 \%$ of all CIC patients reported using some form of OTC laxative, using an average of three OTC products prior to consulting an HCP. HCPs reported that they were aware that most CIC patients try a variety of OTC treatments and lifestyle changes before they seek help from an HCP, and believed that most patients try increasing their fiber intake (41\%) and taking stool softeners (33\%). Regarding the initial CIC conversation with their HCP, patients reported that their HCP recommended general diet changes/ home remedies $(57 \%)$, increasing daily activity/exercise $(41 \%)$, continuing on current OTC laxative [with $(15 \%)$ or without $(23 \%)$ dosage change], and initiation of a prescription CIC treatment $(22 \%)$. Only $40 \%$ of respondents were satisfied or completely satisfied with OTC laxatives, with $47 \%$ of respondents reporting that they experienced diarrhea at least some of the time. 

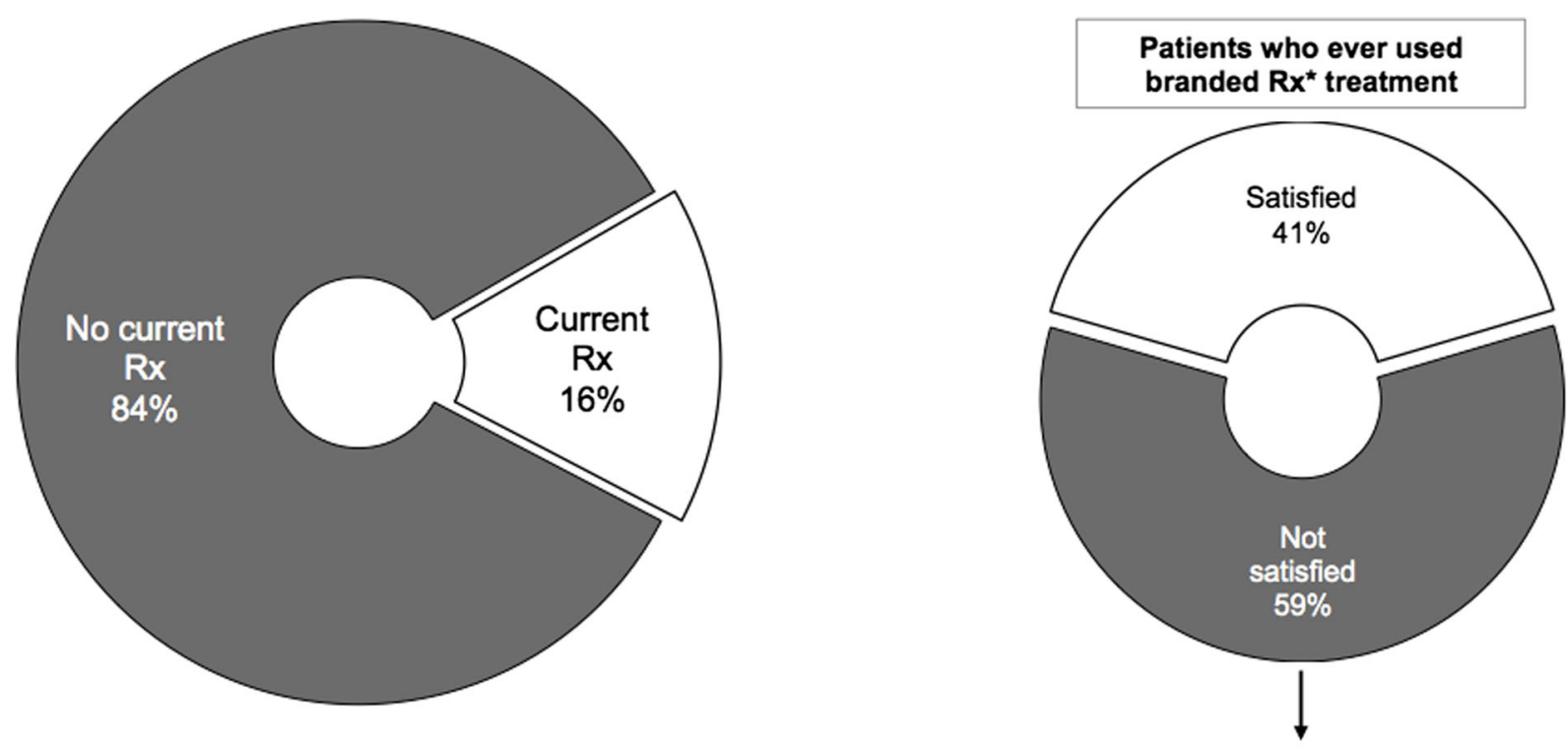

Top reasons not completely satisfied

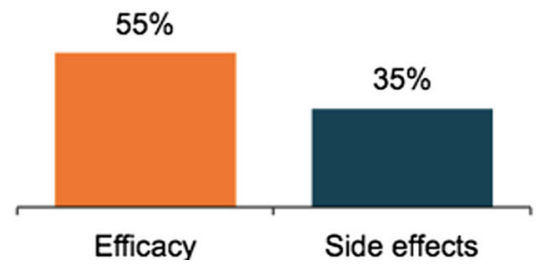

Fig. 1 Experience with current prescription therapies for the treatment of CIC. Left percentages of patients who had ever used any treatment for CIC. Branded Rx* includes linaclotide and lubiprostone. $R x$ prescription

In describing their current treatment plan, only $16 \%$ of patients indicated that they were currently taking a prescription treatment for their CIC symptoms. Less than half (41\%) reported being satisfied or completely satisfied with their branded prescription medication (ordinal scale of 1-7), resulting in 59\% of patients with CIC potentially seeking new treatment options (Fig. 1). The most frequent reasons why current and past users were not completely satisfied with their branded prescription were lack of efficacy (55\%) and presence of side effects (35\%). On average, patients reported that their prescription therapy provided relief of symptoms after 3 days (mean, 2.7 days), with only $26 \%$ reporting that they achieved relief from their CIC symptoms within 1 day. In contrast, the majority of all patients (57\%) agreed that they expected their prescription treatment should work within $24 \mathrm{~h}$. Of patients who initiated treatment with a prescription therapy and received relief within
$24 \mathrm{~h}, 74 \%$ reported satisfaction with the time that the treatment took to work. Overall satisfaction with time to relief was higher among patients who were taking prescription treatments for their CIC symptoms (56\%) compared to patients taking OTC laxatives (40\%).

Despite using a prescription treatment, $86 \%$ of these patients reported a wide variety of residual CIC symptoms (multiple-choice list). Of those patients still experiencing CIC symptoms despite prescription therapy, the most common remaining CIC symptoms were difficulty with bowel movement and infrequent stools, followed by abdominal symptoms of discomfort and bloating/distension (Table 5).

\section{Challenges to the Management of CIC Symptoms}

Despite the availability of various OTC and prescription therapies, all HCPs indicated that $\mathrm{CIC}$ is a challenging disorder to manage 
Table 5 Symptoms still experienced by patients with CIC despite current prescription treatment

\begin{tabular}{ll}
\hline Symptoms & $\begin{array}{l}\text { CIC patients } \\
n=218 ~(\%)\end{array}$ \\
\hline Difficulty with bowel movement & 35 \\
Infrequent stools & 30 \\
Abdominal discomfort & 29 \\
Bloating/Distension & 27 \\
Straining & 26 \\
Feeling of incomplete bowel & 26 \\
$\quad$ movement & 21 \\
Hard, lumpy, or pebble-like stools & 13 \\
Stomach cramps & 9 \\
Nausea & 4 \\
Bleeding (blood on stool, toilet paper, & \\
$\quad$ or in toilet bowl) & 4 \\
Rectal pain & \\
\hline
\end{tabular}

${ }^{a}$ Patients who currently used prescription CIC treatment and whose constipation symptoms were not relieved all the time with that prescription

(Table 6). Only $22 \%$ of HCPs reported being satisfied or completely satisfied with current prescription treatments, with $78 \%$ feeling that there is room for improvement. Inadequate treatment response rates $(55 \%)$ and patient adherence or compliance (55\%) were the most common challenges reported (multiple-choice list), followed by the lack of CIC-specific treatment options (34\%) and the management of treatment-emergent diarrhea (34\%). Of patients with CIC who discontinued their prescription treatment, $53 \%$ did so because of treatment-emergent diarrhea and $36 \%$ did so because of lack of efficacy. One-quarter (28\%) of CIC patients reported experiencing diarrhea while taking an OTC laxative or prescription treatment, with this proportion increasing to $34 \%$ in patients with more severe CIC. Most patients (70\%) did not agree with the statement that diarrhea is an acceptable outcome of taking a medication to relieve symptoms of constipation, while $40 \%$ agreed with the statement that diarrhea is a sign that my medication(s) is/are working. Only $16 \%$ of HCPs believed that diarrhea was a sign that the CIC treatment was working, with $89 \%$ of HCPs expressing that diarrhea was not an acceptable outcome of treatment. Furthermore, HCPs indicated that they believed that diarrhea prevents patients from enjoying daily activities (42\%), causes patients to travel less (40\%), and results in embarrassment due to having to use the bathroom frequently (37\%).

\section{DISCUSSION}

The BURDEN-CIC study is one of the largest patient-based studies conducted to understand the management pathway, overall satisfaction with care, and unmet treatment needs of patients with CIC. In addition, BURDEN-CIC is the first study of its kind to compare and contrast the opinions of HCPs with those of individuals with CIC so as to more fully understand the care pathway, treatment pattern, and attitudes about available treatments for patients diagnosed with CIC in the US. While CIC respondents and HCPs demonstrated alignment regarding the CIC symptom bothersomeness and impact on activities of daily living and quality of life, as well as dissatisfaction with current CIC treatments, there were also areas in which patients and HCPs diverged in their perceptions of CIC.

The findings from the BURDEN-CIC study are consistent with those reported previously regarding the bothersomeness of CIC symptoms and the overall burden on daily activities, as well as the low levels of satisfaction and high degrees of frustration with currently available treatment options for CIC $[4,9,10]$. Of the BURDEN-CIC respondents, $40 \%$ were satisfied or completely satisfied with OTC laxatives and only $56 \%$ of patients reported being satisfied or completely satisfied with their current prescription therapy, citing inadequate efficacy and general side effects as top reasons for not being satisfied. As early as 2007, Johanson et al. [4] found that $47 \%$ of CIC patients were not completely satisfied with their current CIC treatment, with $82 \%$ citing efficacy and $16 \%$ citing adverse effect concerns as the reasons for 
Table 6 Greatest perceived challenges by HCPs to the management of patients with CIC

\begin{tabular}{lllll}
\hline & GE $n=\mathbf{1 5 5}(\%)$ & PCP $\boldsymbol{n}=\mathbf{7 6}(\%)$ & NP $\boldsymbol{n}=\mathbf{5 0}(\%)$ & $\mathbf{P A} \boldsymbol{n}=\mathbf{5 0}(\%)$ \\
\hline $\begin{array}{l}\text { Inadequate response rates to currently available } \\
\quad 64\end{array}$ & 54 & 44 & 40 \\
$\quad$ treatment(s) for CIC & 52 & 50 & 58 & 70 \\
Patient adherence/non-compliance issues & 40 & 28 & 38 & 20 \\
Lack of treatment options for CIC & 36 & 32 & 32 & 36 \\
Managing treatment-related diarrhea & 29 & 21 & 22 & 24 \\
$\begin{array}{l}\text { Managing treatment-related side effects other } \\
\text { than diarrhea }\end{array}$ & 23 & 33 & 38 & 18 \\
Lack of treatment guidelines & & & \\
\hline
\end{tabular}

$G E$ gastroenterologist, $N P$ nurse practitioner, $P A$ physician assistant, $P C P$ primary care physician

dissatisfaction. In an international survey of laxative satisfaction [10], only $28 \%$ of CIC respondents were satisfied or very satisfied with their current laxative and 28\% reported being dissatisfied or very dissatisfied. This survey also found that there was no relationship between laxative type and degree of satisfaction [10]. Despite therapeutic advances with newer medications, new information from BURDEN-CIC identified that both patients and HCPs still have a high level of dissatisfaction with the level of relief provided with fiber, OTC laxatives, and prescription therapies.

Although constipation has been traditionally viewed and managed according to infrequency of bowel movements, the BURDEN-CIC study highlights that CIC is a chronic, multi-symptom disorder with a high level of patient burden requiring an individualized approach to patient management. Respondents entering BURDEN-CIC were heterogeneous as indicated by a wide range of clinical symptoms with varying degrees of severity and burden. In the CIC survey by Johanson et al. [4], more than three-quarters $(76 \%)$ of $\mathrm{CIC}$ respondents rated their constipation as somewhat, very, or extremely bothersome, in comparison to $61 \%$ of patients in this study. Heidelbaugh et al. [9] found that constipation was rated as very or extremely bothersome in $62 \%$ of CIC patients with abdominal symptoms and $40 \%$ of CIC patients without abdominal symptoms. The CIC symptoms most commonly reported as very or extremely bothersome from the
Johanson et al. survey included bloating, straining, hard stool consistency, and infrequent bowel movement [4], whereas the top three most bothersome symptoms reported in BURDEN-CIC were difficulty with bowel movement, straining, and abdominal pain/discomfort. When respondents were asked to rank the single most bothersome symptom, the same order was observed: difficulty with bowel movement (25\%), straining (13\%), and abdominal pain (11\%). These data adhere to the acknowledgement by the Rome Committee that CIC and IBS-C reside on a continuum, and those $11 \%$, while still fitting the diagnostic criteria for CIC, may reside closer to IBS-C on the spectrum. These results underscore the notion that CIC is a heterogeneous condition, with the bothersomeness of symptoms relying primarily on patient perception. While patients categorized here as having more severe CIC may have reported a higher intensity in symptom burden and impairment in activities of daily living, the overall therapeutic approach to patient management did not vary considerably.

Across several studies examining the impact of CIC on quality of life, a quantifiable negative impact of CIC symptomatology on workplace and school productivity has been consistently demonstrated. Johanson et al. [4] found that $73 \%$ of respondents reported that CIC symptoms impacted personal activities, and 69\% reported some degree of productivity impairment, with work or school missed on an average of 2.4 days/month. Interestingly, Heidelbaugh 
et al. [9] found that productivity was disrupted an average of 3.2 days/month in CIC patients with abdominal symptoms resulting in $\sim 1$ day/month of work or school missed due to CIC symptoms. In the BURDEN-CIC study, $60 \%$ of respondents reported that CIC symptoms impacted personal activities at least 1 day/month. In addition, $25 \%$ of respondents in BURDEN-CIC reported that they missed $\sim 60$ days per year of work or school (5 days/month). Lastly, 14\% of CIC respondents reported that they had visited the ER at least once in the past year, which may seem like a relatively high number [12-14], however, this percentage is in close alignment with the $16 \%$ of patients that the GEs in this study who regularly treat CIC patients believed visited the ER for constipation-related symptoms.

Patients and HCPs alike expressed a high level of frustration and stress with the inability of current OTC laxatives and prescription therapies to provide symptomatic relief, but acknowledged that this was a consequence of having to manage the disorder appropriately and saw no real alternative. Further, where symptomatic relief was achieved with prescription treatment options, the side effect profile of these agents (primarily diarrhea) had a significant impact on activities of daily living and, consequently, treatment satisfaction. Indeed, both HCPs and patients were aligned in their attitudes of treatment-related diarrhea, with the majority of HCPs (89\%) and patients (70\%) expressing that diarrhea was not an acceptable treatment outcome. Of particular interest was the direct correlation observed between patients and HCPs in relation to the bothersomeness and the prevalence of individual CIC symptoms, as well as the correlation between the psychological toll, impact on quality of life, and the degree of disease severity.

Although the BURDEN-CIC study identified a high level of dissatisfaction among patients and HCPs with current OTC and prescription treatment options, the results may be limited by the study design. The population of HCPs, though screened for experience in the management of patients with CIC, may be self-selected based on study participation interest and were not necessarily the HCPs who treated the patient respondents. Finally, respondents with CIC were identified as having CIC either based on patient report or based on a screening questionnaire using Rome IV criteria.

\section{CONCLUSION}

The BURDEN-CIC study is the first large-scale patient and HCP survey to highlight the frustration and high level of dissatisfaction with OTC laxatives and prescription therapies in the management of CIC. Despite this awareness of a heightened sense of frustration for individuals suffering from CIC, there was not a complete alignment between patients and HCPs regarding overall treatment outcomes. While HCPs expressed dissatisfaction with overall expectations of treatments, many patients have become accepting of the physical and social limitations of the disorder. The notion that patients are accepting of CIC as part of their daily life whereas HCPs believe patients are obsessed with CIC symptoms is an insight that may be a subtle yet important attitudinal disconnect between patients and their healthcare providers. HCPs also expressed dissatisfaction with the efficacy of current treatments-especially in regard to treatment-related diarrhea-whereas many patients felt that there was little else that could be done to help manage their condition. Interestingly, only $16 \%$ of patients in BURDEN-CIC indicated currently taking a prescription CIC treatment, and in consideration of the high level of dissatisfaction with OTC treatments, another study examining the barriers to prescribing branded medications may be of interest to better understand this poor utilization.

The BURDEN-CIC study emphasizes the unmet needs of CIC patients as well as those of the HCPs who treat these patients. Ongoing dialogue and additional treatment options are needed between HCPs and CIC patients to ensure appropriate treatment expectations are achieved.

\section{ACKNOWLEDGMENTS}

Synergy Pharmaceuticals Inc. provided funding for this study, article processing charges and the 
open access fee. All authors had full access to all the data in this study and take complete responsibility for the integrity of the data and accuracy of the data analysis. Writing and editorial support were provided by Mary Tom, $\mathrm{PhD}$, Nicole Coolbaugh, and Philip Sjostedt, BPharm, of The Medicine Group (New Hope, PA, USA). This support was funded by Synergy Pharmaceuticals Inc. All named authors meet the International Committee of Medical Journal Editors (ICMJE) criteria for authorship for this manuscript, take responsibility for the integrity of the work as a whole, and have given final approval to the version to be published.

Disclosures. Lucinda A. Harris has participated in advisory boards and has served as a consultant for Synergy Pharmaceuticals Inc. Eamonn M.M. Quigley has participated in advisory boards and has served as a consultant for Synergy Pharmaceuticals Inc. Leslie Magnus is an employee and stockholder in Synergy Pharmaceuticals Inc. John Horn and Michele Kissous-Hunt have nothing to disclose.

Compliance with Ethics Guidelines. The patient protocol and associated materials were reviewed and approved by the Western Institutional Review Board. Both the Patient and HCP questionnaires were conducted by GfK according to the globally accepted standards of good clinical practice (as defined in the International Conference on Harmonisation E6 Guideline for Good Clinical Practice), in accordance with the Health Insurance Portability and Accountability Act, and in keeping with local regulations. Informed consent was obtained from all respondents for being included in the study.

Open Access. This article is distributed under the terms of the Creative Commons Attribution-NonCommercial 4.0 International License (http://creativecommons.org/licenses/ by-nc/4.0/), which permits any noncommercial use, distribution, and reproduction in any medium, provided you give appropriate credit to the original author(s) and the source, provide a link to the Creative Commons license, and indicate if changes were made.

\section{REFERENCES}

1. Suares NC, Ford AC. Prevalence of, and risk factors for, chronic idiopathic constipation in the community: systematic review and meta-analysis. Am J Gastroenterol. 2011;106:1582-91.

2. Higgins PD, Johanson JF. Epidemiology of constipation in North America: a systematic review. Am J Gastroenterol. 2004;99:750-9.

3. Lacy BE, Mearin F, Chang L, et al. Bowel disorders. Gastroenterology. 2016;150(1393-407):e5.

4. Johanson JF, Kralstein J. Chronic constipation: a survey of the patient perspective. Aliment Pharmacol Ther. 2007;25:599-608.

5. Nellesen D, Yee K, Chawla A, Lewis BE, Carson RT. A systematic review of the economic and humanistic burden of illness in irritable bowel syndrome and chronic constipation. J Manag Care Spec Pharm. 2013;19:755-64.

6. Pare P, Ferrazzi S, Thompson WG, Irvine EJ, Rance L. An epidemiological survey of constipation in canada: definitions, rates, demographics, and predictors of health care seeking. Am J Gastroenterol. 2001;96:3130-7.

7. Bharucha AE, Pemberton JH, Locke GR 3rd. American Gastroenterological Association technical review on constipation. Gastroenterology. 2013;144:218-38.

8. Emmanuel AV, Tack J, Quigley EM, Talley NJ. Pharmacological management of constipation. Neurogastroenterol Motil. 2009;21(Suppl 2):41-54.

9. Heidelbaugh JJ, Stelwagon M, Miller SA, Shea EP, Chey WD. The spectrum of constipation-predominant irritable bowel syndrome and chronic idiopathic constipation: US survey assessing symptoms, care seeking, and disease burden. Am J Gastroenterol. 2015;110:580-7.

10. Muller-Lissner S, Tack J, Feng Y, Schenck F, Specht Gryp R. Levels of satisfaction with current chronic constipation treatment options in Europe-an internet survey. Aliment Pharmacol Ther. 2013;37:137-45.

11. Knowledge Panel ${ }^{\circledR}$ recruitment and sample survey methodologies. http://www.gfk.com/fileadmin/ user_upload/dyna_content/US/documents/Knowledge Panel_Methodology.pdf. Accessed 25 May 17.

12. Sommers T, Corban C, Sengupta N, et al. Emergency department burden of constipation in the United States from 2006 to 2011. Am J Gastroenterol. 2015;110:572-9. 
13. Corban C, Sommers T, Sengupta N, et al. Fecal impaction in the emergency department: an analysis of frequency and associated charges in 2011. J Clin Gastroenterol. 2016;50:572-7.

14. Myer PA, Mannalithara A, Singh G, Singh G, Pasricha PJ, Ladabaum U. Clinical and economic burden of emergency department visits due to gastrointestinal diseases in the United States. Am J Gastroenterol. 2013;108:1496-507. 